\title{
Multiple Toy Magnets Ingestion; An Alarming Emergency
}

\section{Mukhtar Mehboob*, Manzoor Ahmed, Muhammad Zubair and Rubina \\ Naz}

Mohtarma Shaheed Benazir Bhutto Hospital, Quetta, Pakistan

*Corresponding Author: Mukhtar Mehboob, Mohtarma Shaheed Benazir Bhutto Hospital, Quetta, Pakistan.
Received: April 29, 2021

Published: August 30, 2021

(C) All rights are reserved by Mukhtar

Mehboob., et al.

\begin{abstract}
Ingestion of a foreign body in children inadvertent or intentional is a common emergency worldwide. A 2-year-old girl who ingested three magnets was presented with vomiting and generalized abdominal pain. Plain abdominal X-Ray revealed three oval shape toy magnets attached together in the upper abdomen. Exploratory laparotomy revealed two magnets in the stomach, which were evacuated. The third magnet was removed from $4^{\text {th }}$ part of the duodenum. The perforation in the duodenum and posterior wall of stomach were repaired. The recovery of the patient was smooth. It was evident that body temperature and acidic $\&$ alkaline environment of intestine not hampered the magnetic properties. The magnetic forces exceed the tissue perfusion pressure of intestine lead to local necrosis and perforation.
\end{abstract}

Keywords: Foreign Body; Magnet; Complications

\section{Introduction}

In the Unites States, a total of 94,820 cases were reported in 2015 , and 68,371 of these cases occurred in pediatric patients aged $\leq 5$ years [1]. The literature revealed that children between 6 months to 3 years of age are very much prone to foreign body ingestion. The reported ingested foreign bodies included coins, toys, part of toys, small toy batteries, jewellery, $2 \mathrm{~cm}$ long plastic axe and magnet [2]. Ingestion of magnet has distinct characteristics from other foreign bodies. Single magnet may pass un-noticed from bowel spontaneously without any complication but two or more magnet may pose different complications and needs surgical intervention.

In a report by Macedo M., et al. an 18-month-old girl ingested two magnets developed small bowel intestinal fistula, which were successfully treated by surgery [3]. In another report by Cauchi.
A 9-year-old girl with history of ingestion of 12 small magnets presenting with abdominal pain, vomiting, and diarrhea. Her laparotomy revealed multiple ileal perforations [4]. In our patient of 2- year-old girl, who swallowed the three oval shape magnets presented with signs and symptoms of peritonitis were operated for gut perforation.

In our study the properties of magnet and their hazards in human body was highlighted. Healthcare professionals must be aware of the potential risks and possible complications of magnet ingestion, which should be treated differently from ingestion of other types of objects due to high associated morbidity.

\section{Case Report}

A 2-year-old girl presented in outpatient department with history of vomiting and abdominal pain from last 2 days. Her parents 
states that she vomited 4 times in last 24 hours. The vomitus was greenish with food contents and of moderate amount. The abdominal pain was generalized and she was in distress. Her parents gave the history that she had ingested three toy magnets 5 days back. They consulted with their family doctor, who advised them wait and watch for spontaneous expulsion in faeces. Unfortunately, magnets not expelled and patient become symptomatic. General physical examination revealed that she had pulse 130 beats $/ \mathrm{min}$, temperature $100^{\circ} \mathrm{F}$ and respiratory rate 40 breaths $/ \mathrm{min}$. Systemic examination revealed generalized abdominal tenderness and guarding. Gut sounds was sluggish.

Primary investigations revealed $\mathrm{Hb} 12.5 \mathrm{gm} / \mathrm{dl}$, TLC 16000/ $\mathrm{mm}^{3}$, blood sugar 104gm/dl, $\mathrm{Na}^{+} 142 \mathrm{~m} \mathrm{Eq} / \mathrm{L}, \mathrm{K}^{+} 3.4 \mathrm{~m} \mathrm{Eq} / \mathrm{L}, \mathrm{Cl}^{+}$ $102 \mathrm{~m} \mathrm{Eq/L}$. Plain abdominal X-Ray revealed three oval shape toy magnets in the upper abdomen. Exploratory laparotomy was planned. Inform consent was taken from parents. The abdomen was opened via upper midline incision. There was no peritoneal contamination. Magnets were palpated in the stomach. Stomach was opened near the greater curvature of the stomach where magnet was felt. Two magnets were removed but third were not found there. The search for third was made. It was found in fourth part of the duodenum. There was also a perforation with necrotic edges. The third magnet was removed from there. There was also a hole in the transvers mesocolon and a collection of bile. Lesser omentum was opened through gastro colic ligament. There was a perforation in the posterior wall of the stomach with necrotic edges.

The oval toy magnets have very strong forces of contraction. One of the magnets detached from the two and passed into the duodenum. In the fourth part of the duodenum, which anatomically very close to the stomach. The magnet in the duodenum due to strong forces of attraction attached to the magnets in the stomach with intervening wall of duodenum, transverse mesocolon mesentery and wall of stomach. These intervening tissues due to strong forces of magnetic attraction, which exceeds the tissue perfusion pressure becomes ischemic and finally necrosed. These lead to perforation of posterior wall of the stomach, transverse mesocolon and fourth part of the duodenum. The bile in the lesser omentum was mopped. The margins of perforation of stomach and duodenum was refreshed and repaired. The lesser sac was closed. Abdominal cavity was closed. Postoperative recovery of the patient was uneventful.

\section{Discussion}

There are four types of permanent magnets, Neodymium iron boron, Samarium cobalt, Alnico and ceramic or ferrite magnet. Neodymium iron boron has strong force of attraction. In each variety of magnet the substances are arranged as, diamagnetic (no effect of temperature), paramagnetic (inverse relation with temperature) and ferromagnetic (not exact inverse relation with temperature). The ferromagnetic when heated to high temperature (Curie temperature) it will be converted into paramagnetic. The neodymium magnet will lose its magnetism when heated above its Curie point [5]. Our patient ingested three Neodymium iron boron magnet. They were exposed to internal body temperature. In humans, the average internal temperature is $37.0^{\circ} \mathrm{C}\left(98.6^{\circ} \mathrm{F}\right)$, The stomach likes to keep its core temperature steady at about $100^{\circ} \mathrm{F}$. which is when the best digestion occurs [6]. The maximum recommended curie temperature for the Neodymium iron boron magnets is $310-400^{\circ} \mathrm{C}$ [7]. Our study proved that these magnets curie temperature is more than body core temperature. This was the strong reason that these magnets were attached together and not allowed any magnet to pass through spontaneously but lead to complication.

The effect of strong mineral acids on Neodymium iron boron magnets particles leads to leaching of it and results in release of Neodymium and results in adverse effects in the environment [8]. 
In our patient the magnets were exposed to hydrochloric acid but no leaching and decay was observed. Ingested magnets need to be treated differently from other ingested objects because of a higher associated morbidity. If the magnets are stationary on serial X-rays and signs of obstruction or increasing abdominal pain are present, then operative intervention should be performed to retrieve the magnets to prevent further complications [9]. In our study X-ray abdomen revealed three magnets attached together seen in upper part of abdomen. The magnets were seen as beaded appearance but on exploration we found two magnets were stick together in stomach and one magnet in fourth part of duodenum, this shows that radiologically it is difficult to recognize that all are in single lumen or in adjacent loops of bowel.

In a study Hayek G., et al. the laboratory tests showed a C-reactive protein level of $256 \mathrm{mg}$ per liter and a white-cell count of 19,300 per cubic millimeter, with $90 \%$ neutrophils [2]. In our study $\mathrm{Hb} 12.5 \mathrm{gm} / \mathrm{dl}$, TLC 16000/mm³ $\mathrm{m} \mathrm{Eq} / \mathrm{L}, \mathrm{K}^{+} 3.4 \mathrm{~m} \mathrm{Eq} / \mathrm{L}, \mathrm{Cl}^{-} 102 \mathrm{~m} \mathrm{Eq} / \mathrm{L}$. The initial management to retrieve magnets from the stomach is simple endoscopy or in combination with Roth Net technique [10]. In our patient these methods were not used because patient presented late and she was in peritonitis. The literature revealed that the magnets located in different parts of the gastrointestinal tract can lead to serious complications such as perforation, obstruction, fistula and volvulus. In our patient there were perforations in the stomach and $4^{\text {th }}$ part of duodenum. The perforations were closed. The patient recovery was smooth.

\section{Conclusion}

A patient with multiple magnet ingestion should be closely monitored and early intervention for evacuation should be performed. The body temperature and gastric acidity not hampers the magnetic power. The magnetic power is more than tissue perfusion pressure of stomach.

\section{Bibliography}

1. Mowry JB., et al. "2015 Annual report of the American Association of Poison Control Centers' National Poison Data System (NPDS): 33rd annual report". Clinical Toxicology (Phila) 54 (2016): 924-1109.
2. Hayek G and D'Assignies G. "An unknowingly swallowed inedible toy". The New England Journal of Medicine 369 (2013): 2535.

3. Macedo M., et al. "Intestinal fistula after magnets ingestion". Einstein 11.2 (2013): 234-236.

4. Cauchi JA and Shawis RN. "Multiple magnets ingestion and gastrointestinal morbidity". Archives of Disease in Childhood 87 (2002): 539-540.

5. Buschow KHJ. "Encyclopedia of Materials: Science and Technology”. Elsevier (2001).

6. Bateman DN. "Effects of meal temperature and volume on the emptying of liquid from the human stomach". Journal of Physiology 331 (1982): 461-467.

7. https://e-magnetsuk.com/neodymium_magnets/temperature_ratings.aspx

8. Gergoric M., et al. "Leaching and Recovery of Rare-Earth Elements from Neodymium Magnet Waste Using Organic Acids". Metals 8 (2018): 721.

9. Kabre R., et al. "Hazards complications of multiple ingested magnets; report of four cases". European Journal of Pediatric Surgery 19.3 (2009): 187-189.

10. Yadav A and Feuerstein JD. "A magnet-induced stomach ulcer causing abdominal pain". Annals of Gastroenterology 30 (2017): 464.

\section{Volume 5 Issue 9 September 2021}

(C) All rights are reserved by Mukhtar Mehboob., et al. 